\section{EL CONSEJO FEDERAL DE INVERSIONES EN LA PROVINCIA DEL CHACO: PROYECTOS, DIAGNÓSTICOS Y PLANIFICACIÓN EN LOS MÁRGENES (1958-1983)}

\author{
THE CONSEJO FEDERAL DE INVERSIONES IN THE \\ PROVINCE OF CHACO: PROJECTS, DIAGNOSTICS AND \\ PLANNING IN THE MARGINS (1958-1983)
}

\section{Adrián Alejandro Almirón}

\section{RESUMEN}

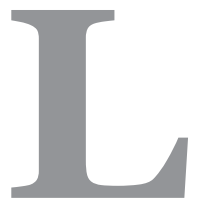

as instituciones técnicas en nuestro país son fundamentales para el estudio de distintos procesos económicos-sociales, en este sentido el Consejo Federal de Inversiones (CFI) representa una de ellas. Su creación en el marco de las ideas que proponía la Comisión Económica para América Latina y el Caribe (CEPAL) permite comprender su importancia y los objetivos que tenía para la región. En este sentido el artículo examina cuales han sido los alcances de los trabajos realizados por el CFI en la provincia del Chaco entre 1958 a 1983, analizando las continuidades y las rupturas que pueden identificarse en las labores realizadas por esta institución. Para la realización de este trabajo analizamos los diversos trabajos publicados por el organismo.

Palabras clave: Chaco, Consejo Federal de Inversiones, planificación.

\section{ABSTRACT}

The technical institutions in our country are fundamental for the study of different economic-social processes; in this sense Consejo Federal de Inversiones (CFI) represents one of them. Its creation within the framework of the ideas proposed by Comisión Económica para America Latina y el Caribe (CEPAL) allows us to understand its importance and the objectives it had for the region. The article examines the scope of the work carried out by the CFI in Chaco between 1958 and 1983, analyzing the continuities and ruptures that can be identified in the work carried out by this institution. To carry out this work we analyze the various works published by the agency.

Keywords: Chaco, Consejo Federal de Inversiones, planning.
Adrián Alejandro Almirón almirón.historia@gmail.com

Facultad de Humanidades

Universidad Nacional del Nordeste ARGENTINA

COMO CITAR ESTE ARTÍCULO Almirón, A. A. (2019). El Consejo Federal de Inversiones en la provincia del Chaco: proyectos, diagnósticos y planificación en los márgenes (19581983). Revista de la Facultad de Ciencias Económicas, 23(2), 15-30.

http://dx.doi.org/10.30972/rfce.2324016

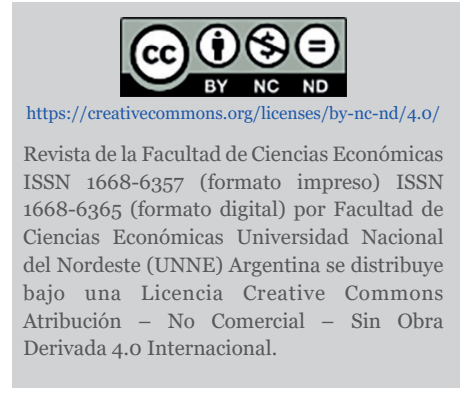




\section{INTRODUCCIÓN}

La política social y económica de nuestro país desde mitad del siglo XX atravesó momentos de tensiones, transformaciones y crisis que fueron acompañadas a partir de una constante modificación las cuales estuvieron ligadas a los cambios de gobierno que ocurrieron desde 1955 en adelante. Este contexto de constante renovación hizo y fueron el origen de diversas medidas que intentaron de resolver las desigualdades y diferencias regionales, en especial en el Nordeste (NEA). De esta manera lo que primara durante este periodo es la acción estatal a partir de un conjunto de medidas y acciones por parte de las instituciones nacionales y provinciales en torno a lograr solucionar diversas situaciones de conflicto.

La trascendencia de este momento histórico radica en los fuertes cambios a nivel político, económico, social y cultural que lo caracterizaron. Estas transformaciones y tensiones se manifestaron en las instituciones que creadas durante este periodo. Como consecuencia desde 1955 a 1983 evidenciamos como se produce dentro del Estado Nacional y provincial diversas estrategias para lograr establecer un nuevo orden social y económico en la región.

Los trabajos académicos hasta el momento han analizado las características que ha tenido la crisis desde una perspectiva económica y social analizando el comportamiento de los distintos actores rurales, desde el estancamiento productivo hasta el proceso de emigración que se da en la región y en Chaco. Desde las instituciones nacionales y provinciales se estableció la necesidad de expandirse en nuevas áreas para fomentar el proceso de ocupación y colonización, estableciendo un nuevo modelo productivo en la provincia, finalizaba el ciclo algodonero y daba una política que implicaba la expansión de cultivos como la soja. Esta situación de transformación implico una readaptación de las instituciones encargadas de promocionar las políticas agrarias e intentar corregir los diferentes conflictos que fueron generándose en el territorio por parte de los actores rurales (Bruniard, 1978; Carlino, 2008; Moglia, 2013; Roze, 2007).

En los últimos años, diversos aportes han propuesto llevar adelante una mirada sobre las prácticas estatales en torno a cómo consolidar la región en el marco de los objetivos propuestos desde la política gubernamental nacional (Bageneta, 2015, Almirón, 2017). Siguiendo esta línea interpretativa, consideramos necesario reconstruir el rol que ha tenido el Consejo Federal de Inversiones en la Provincia del Chaco atendiendo a los objetivos propuestos a nivel nacional y como se han aplicado en la provincia. En este caso nos parece relevante identificar el número de trabajos realizados, los temas a la cuales se dedicaban en sus investigaciones, las recomendaciones y acciones llevadas adelante.

Llevar adelante este estudio nos permitirá comprender cuales han sido las distintas formas en las que el gobierno nacional encontró para realizar el diagnóstico y planificación de las 
políticas públicas. El artículo se encuentra dividido en tres apartados los cuales problematizan por lado el contexto en el cual se crea el Consejo Federal de Inversiones, en una segunda parte llevaremos adelante una descripción sobre qué acciones se realizaron en la provincia a nivel general y en el último apartado realizaremos una descripción sobre los trabajos vinculados a estudios económicos. Como principales fuentes de información se utiliza las memorias del Consejo Federal de Inversiones y las investigaciones realizadas por los expertos para la institución durante el periodo propuesto.

\section{EL PLAN DEL ESTAdo DURANTE LA SEGUNDA MITAD DEL SIGLO XX}

A partir del golpe de Estado de 1955, la política nacional y provincial se verá atravesada por un conjunto de cambios. Fue en este contexto en donde las políticas económicas y sociales fueron puestas en consideración a la manera en que se estaban generando. En este caso, la discusión partía del tipo de intervencionismo que debía realizar el Estado para con la sociedad y en las distintas medidas económicas que se necesitaban.

El desarrollismo con Arturo Frondizi ponía nuevas expectativas a la política económica, la conjunción de estas nuevas expectativas hizo que se generaran en el caso del Chaco situaciones de posibilidades de estudiar cómo se podía avanzar en torno a las políticas fiscales. La ideología desarrollista en lo económico, postulaba la industrialización y el impulso de las industrias básicas, esto permitiría superar los límites impuestos al crecimiento y mejoraría el nivel de vida de los pobladores que se encontraban estancados dada la situación agraria (Belini y Korol, 2012, p.163). Estas acciones respondían y se encontraban en un contexto de revisión sobre el lugar que tenía el Estado como dinamizador de las políticas públicas. Estas reflexiones están vinculadas e inspiradas en los estudios realizados por la Comisión Económica para América Latina y el Caribe (CEPAL) el cual durante esta etapa promovía una activa preocupación en torno a cuales eran las características del subdesarrollo en los distintos países del continente, en el caso de Argentina se caracterizaba por encontrarse el país conformado por distintos niveles de integración al capitalismo. La marginalidad ${ }^{1}$ que constituye a las distintas provincias del NEA es la muestra de las características propias de la inserción de estos espacios subnacionales al capitalismo, siendo estos considerados como subdesarrollados (Rougier, 2004, p.32).

Las discusiones y propuestas se enmarcaron en torno a cómo llevar adelante la política tendiente al despegue y lograr achicar los márgenes de desigualdades que existían en las distintas regiones del país. Una de las estrategias del Estado fue intervenir y planificar a fin de poder acelerar el proceso de desarrollo.

${ }^{1}$ Gino Germani consideraba en 1981 que existían tres tipos de raíces comunes que dieron origen al mismo: 1 . El proceso de extensión de los derechos del hombre; 2 Los procesos de contacto cultural; y 3. La acentuación de los procesos de modernización. 
La planificación económica era entendida por la teoría y por la nueva clase de tecnócratas como un acto arbitrario y a la vez racional, ya que permitirá corregir aquellos aspectos que comportaban elementos irracionales, en definitiva, responsables del atraso y del subdesarrollo (Rougier, 2004, p.35).

Por este motivo, la inversión económica formaba parte del rol del Estado, esto lo convertía en un coordinador político a partir de tres variables: 1. la promoción de la industrialización por sustitución de importaciones, 2. la afirmación del Estado como representante de la Nación, incluyendo a sectores marginales y 3. la racionalización de la intervención activa del Estado en nombre del proyecto de modernización (Lechner 1997, p.8).

Por este motivo, las instituciones con una finalidad de trasformación fueron claves para lograr promover la modernización económica y social. En este caso debemos entender al Estado como "autónomo" entendiendo el mismo a partir de funcionarios, son capaces de diseñan diversas estrategias, no obstante esto no implica que existan tensiones e imposibilidades en su aplicación dentro del Estado (Vigera, 1998). Las instituciones tendrán un rol fundamental en el desarrollo de estas políticas tendientes a la modificación y modernización, aunque debemos considerar lo que plantea Zurbriggen cuando reconoce a las instituciones como espacios de tensión, las instituciones por los diferentes actores (2006, p.76).

En este caso, la conformación de nuevas estrategias delineadas por el gobierno nacional y provincial desde la clase política desde la autonomía propia estatal, confrontaba con los propósitos de los distintos actores que se encontraban en la región chaqueña. Todas estas acciones del Estado con sus cuerpos de técnicos nos demuestran el esfuerzo por lograr una planificación, es decir el esfuerzo organizador de la voluntad política que se produce en el ámbito del gobierno y el cual se evidencia en la forma operativa que tiene el Estado en torno a la administración para lograr y materializar la política pública (Bernaza, 2006). En este sentido el Consejo Federal de Inversiones es una muestra clave de estos objetivos y propuestas por parte del Estado, sus expertos serán los representantes directos del Estado quienes con sus informes no solo expondrán una realidad sino que además demuestran un capital simbólico, el cual les permite proponer y diseñar como operar sobre esa realidad, es decir la política pública² $^{2}$ (Bourdieu, 1997, p.123). Su funcionamiento junto con otras instituciones, permiten comprender los objetivos del Estado junto con las propuestas realizadas en el marco de un contexto socio-económico de la provincia.

\footnotetext{
${ }^{2}$ Bourdieu Pierre describía este tipo de capital simbólico con los expertos de la siguiente forma: se llama comisión -conjunto de personas que están investidas de una misión de interés general y requeridas para trascender sus intereses particulares para producir proposiciones universales-, los personajes oficiales han de obrar siempre cuando no sacrificando su punto de vista particular «al punto 123 de vista de la sociedad», por lo menos constituyendo su punto de vista en punto de vista legítimo, es decir universal, en particular mediante el recurso a una retórica de lo oficial. (1997, p.123).
} 


\section{EL CONSEJO FEDERAL DE INVERSIONES EN CHACO: DEL DIAGNÓSTICO A LA PLANIFICACIÓN}

El Consejo Federal de Inversiones (de ahora en más CFI) se creó en 1959 con la intención de poder lograr promover el desarrollo de la economía en las distintas regiones del país. El CFI nace con el objetivo lograr brindar herramientas conceptuales e instrumentos políticos para lograr que todos los Estados provinciales pudieran acceder al progreso, mediante la articulación de las políticas públicas, en este sentido el rol del Estado como promotor de las políticas fue esencial a la hora de lograr modificaciones de estructura en el país. En su artículo 1 describe el objetivo de la institución "cómo organismo permanente de investigación, coordinación y asesoramiento, encargado de recomendar las medidas necesarias para una adecuada política de inversiones y mejor utilización de los distintos medios económicos contundentes al logro de un desarrollo basado en la descentralización". Todas las provincias eran beneficiadas por este organismo, pero previamente debería autorizarse su funcionamiento.

El gobernador Anselmo Duca en 1959 solicitaba a la Cámara de Diputados de la provincia del Chaco, la ratificación del organismo para que pueda funcionar, la misma logra el reconocimiento como personaría jurídica de derecho privado, trabajando en la provincia desde este momento con diversos estudios y proyectos tendientes al desarrollo.

En 1961, Frondizi crea el Consejo Nacional de Desarrollo (en adelante CONADE) con el objetivo de afianzar la planificación de la económica a partir de una institución que pudiera brinda un conocimiento cabal y pudiera además proponer diversas estrategias de acción para lograr una mejora en la estructura económica del país. Asimismo se encargaría de reunir la financiación externa para poder dinamizar las obras publicas y el desarrollo de la industria (Jáuregui, 2013). Teniendo ambas instituciones el objetivo de planificar nuevas políticas económicas. No obstante, Rougier (2004, p.59) sostiene que los informes de ambos consejos, fueron elaborados con una creciente sofisticación técnica, pero con una escasa posibilidad de implementación y en muchos casos con mínima vinculación con la economía real.

Pese a esta observación el CFI entre 1963 y 1983 realizó más de 57 trabajos de llevados en distintas áreas para la provincia del Chaco. Los mismo fueron variando de acuerdo a la temática y área que se encargaba, mayormente lo que evidenciamos en este sentido es la presencia de estudios referidos a la proyección de obras públicas y asesoramiento a la provincia con estudios referidos a la conformación de la mejora institucional, como ser cursos de capacitación al personal técnico o en formas de llevar adelante mejoras en la manera de administrar los recursos. Estos informes nos permiten comprender como lentamente el gobierno provincial se estaba constituyendo y organizando en distintas áreas. Quienes realizaban estos informes eran distintos expertos entre los cuales se destacaban economistas, sociólogos, geógrafos, antropólogos, historiadores, ingenieros que han logrado hacer una descripción en torno a cuáles eran los problemas que debían solucionar y atender la provincia para producir el despegue económico. 
Hacia 1968 en Chaco, se llevó adelante un informe sobre la estructura del Ministerio de Agricultura y Ganadería, en el mismo se destacaba la necesidad de las potencialidades que tenían con sus recursos humanos pero también ponía en balance los aspectos que debía resolver para lograr los indicativos en torno a una mejor gestión de los recursos. Hacia 1973 se llevaron adelante distintos estudios de distintos tipos, destacándose los siguientes trabajos: posibilidad de industrialización del sorgo granifero, la cooperación técnica en producción lechera, el estudio de factibilidad de la instalación de una o más curtiembre, la obtención de resina fenólicas, el estudio para la instalación de un frigorífico, la comercialización de maderas y el mercado de artesanías regionales.

Un año después se llevó adelante un contrato en donde el gobierno provincial solicitaba la colaboración técnica en el centro de informática de la Provincia para las áreas de la estructura de gobierno, en sus comentarios se destacan las observaciones sobre el personal que se encontraban trabajando y sus prácticas:

Nos encontramos con una acentuada falta de producción y un bajo nivel de rendimiento, tomando como indicador los resultados obtenidos.

Las bajas remuneraciones originan en el personal, un estado de continua discordia, hacia toda medida de tipo laboral y organizativa; a su vez, esto genera poca dedicación, la que se hace notar en la escasa iniciativa y poca laboriosidad (...). El hecho de que la casi totalidad de los agentes sean estudiantes universitarios, no es ningún atributo de tipo positivo, ya que los permisos que legalmente se deben conceder afectan notablemente la producción. En fechas de exámenes, hemos tenido una inasistencia de un 70\% del personal (...). El panorama que presenta toda nuestra actividad a desarrollar, no es del todo alentador, ya que se debería solucionar que escapa a nuestras posibilidades (CFI, 1974, p.3).

De esta manera la descripción realizada por estos técnicos eran observaciones críticas en torno al funcionamiento institucional, aunque las mismas no fueron atendidas por el gobierno provincial de manera inmediata. No obstante es importante destacar la funcionalidad del CFI como un organismo asesor frente a diversos temas. Tal fue su centralidad en la creación del funcionamiento provincial, que brindaron asesoramiento para la creación de la empresa de computación del Estado en 1977, una sociedad anónima con un porcentaje mayoritario de capital estatal, "ECOM", este surge a partir de brindar prestación de servicios de sistematización y procesamientos de datos. Otro estudio muy relevante para el desarrollo de la región fue el estudio del puente Chaco-Corrientes con observaciones en torno a cómo debía proyectarse y emplazarse la obra. 
No obstante, además de estas consideraciones en torno a estas cuestiones, los aportes en vinculación a cómo debía realizarse las mejoras en el plano institucional, nos parece muy interesante los estudios que el CFI llevó delante de la cuestión económicas en el Chaco. En el marco de la política de reconversión que comienza a ser cada vez más visible e importante de aplicar para el cambio en la productividad, los estudios técnicos tuvieron y fueron el fundamento de la generación de políticas públicas que irán lentamente aplicándose.

\section{ESTUDIOS ECONÓMICOS}

El primer estudio significativo que nos describe como se encontraba la provincia fue realizado en 1964, como principal fuente de información se basó en datos brindados por los censos de 1947 y 1960. Este trabajo fue titulado como Tenencia de la Tierra y fue es un estudio que permite comprender a nivel nacional como se encontraba el campo argentino. En el caso de la zona chaqueña el estudio rescata no solamente la cuestión de la estructura productiva sino también fue una manera de conocer cómo se encontraba la realidad chaqueña en distintos niveles, salud, educación ${ }^{3}$, poblamiento. En cuanto a los estudios agrarios, el mismo permitió reflexionar sobre un conjunto de realidades que demostraban el grado de marginalidad de la región. El principal inconveniente de atraso identificado fue le la persistencia del monocultivo algodonero, las apreciaciones en torno a esta realidad eran:

Falta de conocimientos de otros cultivos que permiten efectuar racional rotación, tendiente al mantenimiento de la fertilidad del suelo, brindándole ingresos por lo menos similares a los obtenidos por el cultivo del algodón, con la misma regularidad periódica, agudizan los resultados negativos que tal práctica implica. Y, aun en el caso supuesto de conocimientos necesarios como para abandonar el monocultivo, por una explotación más intensiva, con mayor requerimiento de inversiones, juega un rol negativo la precariedad de la tenencia. Precariedad que se agudiza en el caso de los predios involucrados en el sistema subfamiliar, con evidente predominio de tenores de tierras fiscales. Es decir, esta precariedad se traduce en una limitación del mejoramiento de la explotación (CFI, 1964, p.189).

En general este tipo de estudios intentaban promover un uso racional del suelo y luego establecer estrategias para lograr un desarrollo óptimo de los recursos.

\footnotetext{
${ }_{3}^{3}$ Menciona en cuanto a educación: El panorama que presenta la población chaqueña en el plano educacional es desolador. Si bien es cierto que la ciudad de Resistencia es hoy sede de la Universidad del Nordeste y funcionan en ella algunas escuelas y facultades, su reciente creación impide que pueda observarse el efecto de su acción en la formación de los cuadros de la vida regional. Ciencias Sociales cuenta con 398 estudiantes, Humanidades 234, Ciencias Exactas, Físicas y Naturales con 171, Ingeniería 113, Abogacía y Medicina que funcionan en Corrientes, cuenta con millares de estudiantes. CFI. Tenencia de la tierra. P. 185.
} 
Por otro lado el CFI, también tuvo como objetivo comprender a los distintos actores que se encontraban en la provincia, tanto los más representativos a nivel económico como así también los sectores subalternos. En este sentido es interesante el trabajo de campo realizado por un grupo de investigadores del Instituto Guido Di Tella sobre las comunidades indígenas. El estudio se titulo La situación de la población aborigen de la provincia del Chaco y política para su integración a la comunidad nacional, realizado en 1970 el mismo tenía como objetivo llevar adelante una mirada antropológica-sociológica desde una mirada diacrónica como sincrónica. El trabajo de campo permitió visibilizar una marginalidad estructural de las comunidades y se proponían solucionar la realidad en la cual se encontraban padeciendo los indígenas.

La investigación propuesta aquí está dirigida a clarificar las relaciones existentes entre un número limitado de variables. Algunas de estas variables son consideradas teniendo como unidades de análisis los grupos étnicos específicos, mientras que otras variables consideran como unidades de análisis la estructura y el contexto en el cual están situándose, social y especialmente, los indígenas.

Las variables que tienen como unidad de análisis a los grupos indígenas son: 1. organización social y 2. formas culturales. Las variables estructural-contextuales abarcan: 1. vinculación al mercado de trabajo, 2. contexto ecológico y 3. nivel de vida (CFI, 1970, p.2).

Todos los expertos de las ciencias sociales ${ }^{4}$ parten del concepto de integración de las comunidades, esto implicaba que el propósito primordial de estos estudios además fue encontrar herramientas y procedimientos que permitieran a la población indígena poder trabajar, educarse y disponer de diversos recursos. Para lograr este estudio se realizó un trabajo en conjunto con diversas instituciones privadas y públicas de la provincia con la finalidad de poder acceder a información.

La publicación de esta investigación permitió programar y establecer políticas para las comunidades. Otros aportes también estudiaron el desarrollo de las organizaciones rurales la cuales eran prioridad conocer para el gobierno nacional dada la situación de crisis y conflictividad que había comenzado desde 1968 con la aparición de las Ligas Agrarias. En este sentido el informe destaca las formas de representatividad que tenían los mismos en torno a los productores que son estudiados, de esta manera en sus consideraciones sostienen las investigadoras:

Para medir la representatividad de cada una de las asociaciones tratadas no hay que considerar una serie de indicadores que den cuenta de su especificidad frente al conjunto. Para ejemplificar lo dicho se puede comparar a las Sociedades Rurales con las ligas agrarias. En el primer caso, a pesar de tener una baja tasa de afiliación,

\footnotetext{
${ }^{4}$ El equipo estaba integrado por: la directora del proyecto Esther Hermitte. Los antropólogos eran Piedad Batelli, Alejandro Isla, Nicolás Iñigo Carreras. El sociólogo Silva Sigal y Enrique Lubliner. El demógrafo Mario Robirosa, Marĺa Chirico y los economistas Jorge Roulet, Marcelo Cavarozzi.
} 
las sociedades rurales son reconocidas por el conjunto del sector( y por otras organizaciones y grupos sociales de la zona) tanto por la pertenencia de los ganaderos a una clase nacional de tradicional relevancia en la economía argentina como por su participación en organizaciones de notable incidencia en la vida nacional como es la CRA (Confederaciones Rurales Argentinas). Por el contrario, en el caso de las Ligas Agrarias el dato de la afiliación y el caudal de las movilizaciones que lideran constituye un indicador clave para medir su fuerza sindical dado la marginación relativa, política y económica, del sector que agremia (CFI, 1972, p.4).

De esta manera la realidad del campo chaqueño era descrita desde diversos estudios con la finalidad de establecer una construcción del campo como una necesidad de cambiar gran parte de la estructura económica.

El estudio de Daniel Slutzky realizado en 1973 propone una lectura crítica sobre la situación de la tenencia de la tierra desde una perspectiva histórica que a nivel interpretativo logra establecer una secuencia en la ocupación y distribución del suelo. Su estudio "Tenencia y distribución de la tierra en la región NEA" explicaba a partir de ciclos y de etapas la realidad agrícola en Chaco y destacaba nuevamente al monocultivo como un factor particular de la realidad económica de la provincia. Estos estudios sobre la región NEA respondían a la emergencia social y económica que implicaba la realidad nacional, por esto la necesidad de lograr un estudio riguroso en torno a los cuales eran las características de la misma y lo que encontramos en el mismo son estudios desde diversas disciplinas las cuales destacaban estas realidades.

El tercer gobierno de Perón ponía al CFI bajo los objetivos que fueron expresados en $E l$ Plan Trienal para la Reconstrucción Nacional, desde este se intentaba llevar adelante una trasformación del país en distintos niveles y órdenes. El CFI formo parte activa de estos objetivos propuestos desde la dirigencia. En la memoria de la institución en 1974 se destacaba:

Las líneas de trabajo principales han estado en torno de la utilización y aprovechamiento de los recursos provinciales y regionales y de los estudios básicos sobre infraestructuras económico-sociales y recursos humanos, en lo que atañe a las de carácter económico y social. El conjunto de tareas emprendidas, y que retoma una línea original del CFI ha exigido la reconstitución de los equipos necesarios para llevarlas a cabo (...) ha sido retomada también una línea de investigación muy directamente ligada a las relaciones económico-sociales intra e interprovinciales, cual es la de los dos estudios sobre circulación de bienes y cuentas sociales provinciales y regionales. Son aquí analizados los procesos de circulación de bienes, los mecanismos de acumulación y comercialización, así como el armado y preparación de sistemas de contabilidad social provincial y regional (CFI, 1974, p.9). 
Teniendo en cuenta estos objetivos llevados adelante por el CFI, podemos comprender como la política de investigación fue fundamental a la hora de pensar estrategias y propuestas vinculadas a la transformación agraria de la región. Hacia 1974 en la provincia existían proyectos que permitían el desarrollo de las cuencas hidrográficas, el desarrollo de la infraestructura social, la promoción de la actividad industrial, tareas vinculadas a la administración, programación y planificación del Estado junto con investigaciones económicas sociales. Esto hacia un total de 18 trabajos llevados adelante por el organismo. Los estudios que se realizaron durante esta etapa fueron:

- Programación del desarrollo de la cuenca del Río Bermejo

- Estudio de los Bajos Submeriodionales

- Evolución y conformación urbana en la región NEA

- Caracterización socio-económica de las provincias de la Región NEA

- Tenencia y distribución de la tierra en la región NEA

- Las inversiones externas en la Región NEA

- Procesos de transformación, comercialización y financiamiento de los principales productos de la región NEA

- La organización de productores y trabajadores rurales en la Región NEA

- Papel del Estado en el desarrollo de la Región NEA.

De estos estudios mencionados hemos descrito dos de ellos como fundamentales para comprender la realidad agrícola de la década del sesenta y setenta. Sin embargo es interesante además vincular y hacer una lectura de investigaciones que fueron desarrolladas por fuera del CFI pero que estaban vinculadas a los problemas y las soluciones propuestas. Desde la Universidad Nacional del Nordeste se han realizado diversos estudios vinculados sobre la cuestión agraria aportando ideas para promover el despegue de la economía.

La Facultad de Ciencias Agrarias aportó diversos proyectos de investigación que permitieron mejorar y posibilitar cambios en las prácticas culturales en los diversos productores. Desde la Facultad de Ciencias Económicas también tanto las investigaciones como las jornadas de discusión en torno a la situación económica del Chaco permitieron distintas aproximaciones sobre la realidad. Las jornadas en 1970 coorganizada con la Federación Económica del Chaco (FECHACO) posibilitó la reunión de empresarios, corporaciones rurales y funcionarios del gobierno provincial. Este encuentro presento las demandas de los grupos empresariales y la necesidad ejecutar un intervencionismo en la producción y comercialización del algodón por parte del Estado a fin de garantizar las ganancias, pero además se estableció la necesidad de transformar el monocultivo algodonero de un negocio modesto a transformarlo a una empresa, la transformación del modelo productivo en este caso también requería de una labor planificada y del apoyo técnico de los expertos. 
Por su parte la tesis doctoral de Antonio Besil "Análisis de las causas del actual cambio en la estructura del sector agrícola en la Provincia del Chaco" publicada en 1969 brindaba una profunda reflexión del estado del campo chaqueño, en sus conclusiones sobre lo que se requería para el campo exponía la necesidad de afianzar la intervención del apoyo de los técnicos a la hora de establecer la mejora en la productividad:

Con respecto al algodón debe adoptarse, en opinión de INTA Sáenz Peña, un programa que tienda al mejoramiento de la eficiencia de la producción, que comprenda la planificación de las áreas cultivadas, ecológicamente mejor dotadas, para producir más y mejor calidad; el programa debe comprender también el mejoramiento del desmonte y de la industria textil.

Simultáneamente con este planeamiento se deberá promover el cultivo de un tamaño apropiado, como para que el algodón sea integrante de una empresa agrícola diversificada y facilitar la mecanización del apreciable número de labores culturales para asegurar la obtención de cosechas precoces y de alta calidad (...).

Si esto se logra, y con eficiencia, el Chaco entrará en una nueva etapa de su desarrollo económico, desaparecerá su actual vulnerabilidad y podrá revertir el proceso de emigración como resultado de todo ello (Besil, 1969, p. 120).

Estas ideas en torno a los cambios que se requerían estaban también relacionadas a investigaciones realizadas por Washington Valentín Jones, quien llevo adelante su trabajo de investigación en la Estación Experimental Regional de Sáenz Peña sobre el mercado que disponía la producción algodonera chaqueña. Su experiencia se materializó en una tesis doctoral que tendría gran influencia en la política agraria chaqueña. Tanta fue su relevancia que el gobierno le solicitó la realización de un trabajo sobre el estado de situación en que se encontraba el agro, el cual se tituló "Política de tierras, diversificación agropecuaria y crecimiento económico en la provincia del Chaco", publicado en 1966 y también del mismo año Plan de ordenamiento y transformación para el Chaco el cual fue elaborado para la estación experimental de Sáenz Peña. Hacia 1973 realiza un informe para el CFI titulado Industrialización del sorgo en la provincia del Chaco, donde exponía la situación y el avance del sorgo como una posibilidad de crecimiento de la economía frente a la crisis del cultivo algodonero.

Aunque a mediados de la década del 60 se disponía de estudios que señalaban con fundamentación científico-técnica, la situación vigente y pronosticaban cuantitativa y cualitativamente el futuro de la economía algodonera argentina y, especialmente, de la provincia del Chaco, el poder económico y la negligencia del Estado federal hicieron desoír y desoyeron tales advertencias y aconteció lo 
pronosticado en aquellos estudios, ya que el análisis histórico de los hechos posteriores confirmo el pronóstico (Jones, 1973, p.6).

De esta manera la reconversión del Chaco era una necesidad que todos los expertos marcaban para iniciar una nueva etapa. Vinculado a esto la realización de obras públicas para mejorar la calidad del suelo y obtener nuevas áreas de cultivo o de pastoreo fueron fundamentales. En este caso el CFI participó activamente en la elaboración de los informes técnicos y la planificación de las obras de los Bajos Submeridionales. El objetivo era recuperar áreas propensas a inundaciones y sequias para disponerlas y contar con ellas para su trabajo, se estimaba incorporar unos 10 millones de hectáreas entre las provincias de Santiago del Estero, Santa Fe y Chaco, de estas se calculaba que correspondía unos 4 millones de hectáreas, estos estudios se habían iniciado en 1973. En el estudio se consideraba los siguientes puntos:

Un vasto plan de desarrollo integral que comprender obras de saneamiento, infraestructura regional, incorporación de tecnologías optimas adecuadas a las condiciones propias del medio en los establecimientos agropecuarios, como así también, el desarrollo de un servicio de extensión agropecuaria (CFI, 1973, p.2).

Iniciada la última dictadura cívico-militar (1976-1983) se realizaron nuevos estudios a pedido del gobernador de Facto, en este caso el CFI en su estudio de 1978 ponía en cuestión la viabilidad de la obra tal como fue realizada en 1973 y propone ampliar los estudios antes de llevar adelante obras que pudieran implicar inconvenientes en un futuro ${ }^{5}$. Se crea una comisión de coordinación para la ejecución del Plan en donde los gobernadores tenían atribuciones en torno a la aplicación y ejecución de las obras. De acuerdo a lo normativo, la comisión podía disponer en buscar las herramientas para la ejecución de obras e investigación para fomentar y consolidar la región, tal como lo sostiene el informe:

El desarrollo del Plan es un objetivo de largo plazo lo cual no supone que los estudios de investigaciones que se proponen sea una tarea para el futuro. Todo lo contrario, el objetivo señalado será el resultado de la suma de estudios e investigaciones que se realicen en plazos cortos (CFI, 1978, p.12).

En Chaco se realizaron trabajos en el sur de la provincia principalmente en la zona de Santa Silvina con diques de contención. Terminada la última dictadura cívico-militar (1976-1983), el CFI con el regreso de la democracia inicia nuevamente los estudios sobre la región desde diversas perspectivas.

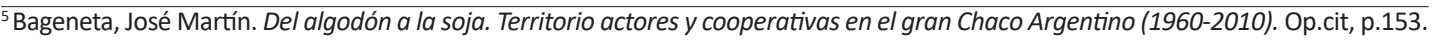


De esta manera la experiencia de aportes teóricos y metodológicos sobre cómo mejorar la productividad y la región fueron notables para conocer y reflexionar sobre continuidades en torno a la política estatal durante este periodo, más allá de las modificaciones en lo político, la persistencia de determinadas instituciones y la continuidad en los mismos trabajos nos permiten comprender cierta regularidad en intentar solucionar problemas estructurales.

\section{CONSIDERACIONES FINALES}

Desde la segunda mitad del siglo XX en nuestro país el debate sobre el rol del Estado y las propuestas sobre como analizar y combatir las desigualdades en las diversas regiones. Las teorías de la CEPAL fueron las que orientaron la mirada y las estrategias llevadas adelante por los distintos gobiernos nacionales en Latinoamerica y en el interior de cada uno de ellas.

En el caso de Argentina el CFI responde a este contexto, su surgimiento permitió en poco tiempo lograr realizar diagnósticos sobre las realidades económicas y sociales, las cuales hasta ese momento se tenían registros fragmentados esparcidos en distintos informes. Estos análisis permitieron en un primero momento tener un diagnóstico de la situación de estancamiento y subdesarrollo por la cual el interior del país se encontraba atravesando. El paso siguiente de esta etapa fue lograr construir una planificación de acción estatal para revertir tales situaciones, en el caso de Chaco la más representativa fueron los trabajos realizados en los Bajos Submeridionales, las políticas sobre el cultivo de soja que fueron desde la década del setenta en aumento, las cuales permitían y lograban construir un nuevo escenario agrario en la provincia.

En este sentido la importancia del CFI nos permite comprender su acción relevante como representante directa de la autonomía estatal y el fortalecimiento institucional el cual representa una política de largo plazo, es por ello que podemos comprender su funcionamiento más allá de las rupturas políticas, estas instituciones permanecieron no lejos de tensiones y de conflictos dentro de su funcionamiento, pero han llevado adelante trabajos de campo y de gabinete que pueden comprenderse desde una continuidad.

De esta manera la labor profesional del CFI durante estos primeros años de institución, nos demuestran los esfuerzos hechos por el conjunto de expertos en lograr brindar herramientas y propuestas para cambiar diversos aspectos que nos caracterizaban como una provincia marginal. 


\section{REFERENCIAS BIBLIOGRÁFICAS}

Almirón, A. A. (2017). Los expertos en el chaco: investigación y política agraria (1920-1972). Revista Coordenadas. Revista de Historia local y regional. Año IV, Número 1, enero-junio 2017. Recuperado de: http://ppct.caicyt.gov.ar/index.php/coordenadas/article/view/11217/pdf

Almirón, A. A. (2018). Política de tierra y colonización para las comunidades indígenas de la provincia del Chaco: proyectos, reclamos y regularización de la ocupación (1951-1987). Revista Res Gesta. $N^{\circ} 54$. Facultad de Derecho y Ciencias Sociales de Rosario. Recuperado de: http://erevistas.uca.edu.ar/index.php/RGES/article/view/1316/1342

Almirón, A. A. (2011). Comentario bibliográfico. Ruffini, Martha y Blacha, Luis (eds.): Burocracia, tecnología y agro en espacios marginales. Rosario: Prohistoria.

Bageneta, J. M. (2015). Del algodón a la soja. Territorio actores y cooperativas en el gran Chaco Argentino (1960-2010). Buenos Aires: INTERCOP.

Bageneta, J. M. (2015). Jones Valentín Howell Washington. Una trayectoria intelectual y las transformaciones del algodón. Posadas: La Rivada. Recuperado de: http://www.larivada. com.ar/index.php/ediciones-anteriores/65-numero-5-diciembre-2015/articulos-5/110-unatrayectoria-intelectual

Belini, C. \& Korol, J. C. (2012). Historia económica de la Argentina en el siglo XX. Buenos Aires: Editorial Siglo XXI.

Besil, A. C. (1969). Análisis de las causas del actual cambio en la estructura del sector agrícola en la Provincia del Chaco. Argentina: UNNE.

Bernazza, C. A. (2006). La planificación gubernamental en Argentina. Experiencias del período 1974-200o como puntos de partida hacia un nuevo paradigma. Argentina: Flacso.

Brodhersohn, V., Slutzky D. \& Valenzuela C. (2009). Dependencia interna y desarrollo: el caso del Chaco. Resistencia: Editorial La Paz.

Bruniard, E. y colaboradores. (1978). El Gran Chaco Argentino: ensayo de interpretación geográfica, 1975-1978. Instituto de Geografía de la Facultad de Humanidades. Resistencia, Chaco, Argentina. 
Bourdieu, P. (1997). Razones prácticas. Sobre la teoría de la acción. Barcelona: Anagrama.

Carlino, A. (2008). Economía provincial y financiamiento público: el Banco de la Provincia del Chaco: 1956-1983. Buenos Aires: Cooperativas CFI (1973). Bajo Submeridionales. Buenos Aires.

CFI. (1978). Estudio integral de los bajos submeridionales, T. VII, Buenos Aires.

CFI. (1974). Informe sobre centro de informática de la Administración pública de la provincia del Chaco. Resistencia, 20 de septiembre de 1974.

CFI. (1974). Memoria Anual de 1974. Buenos Aires.

CFI. (1970). Situación de la población aborigen de la provincia del Chaco y política para su integración a la comunidad nacional. Diciembre 1970, p.2.

CFI. (1964). Tenencia de la tierra. Buenos Aires.

CFI. Golbert L. \& Lucchini de Timerman C. (1972). Respuesta a las observaciones por el CFI al primer informe parcial. La organización de los productores rurales del NEA. Buenos Aires.

CFI. Jones, V. \& Suñer L. (1973). Industrialización del sorgo en la provincia del Chaco. Rosario.

Germani, G. (1980). El concepto de marginalidad. Buenos Aires: Nueva Visión.

Girbal-Blacha, N. (2011). Vivir en los márgenes Estado, políticas públicas y conflictos sociales. El gran Chaco Argentino en la primera mitad del siglo XX. Rosario: Prohistoria.

Jáuregui, A. (2013). La planificación en la Argentina: el CONADE y el PND (1960-1966). Anuario del Centro de Estudios Históricos "Prof. Carlos Segreti” No 13, Año 13. Córdoba, 2013, pp. 243-266.

Jones, V. H. W. (1967). Política de tierras, diversificación agropecuaria y crecimiento en la provincia del Chaco. Resistencia: Ministerio de Agricultura y Ganadería del Chaco. 
Lattuada, M. (1988). Política agraria y partidos políticos (1946-1983). Buenos Aires: Centro Editor de América Latina.

Lechner, N. (2003). Tres Formas de Coordinación Social. Nueva Época, 1(2),

Miranda, G. (2007). Los tres ciclos Chaqueños. Chaco: Ed. La Paz.

Moglia, L. (2013). El cooperativismo agrícola en la agenda pública de una nueva provincia (1951-1962). Blacha Luis y Poggi Marina (comp.). Redes y representaciones en el poder rural. Rosario: La Quinta Pata \& Camino Ediciones.

Rougier, M. (2004). Industria, finanzas e instituciones en la Argentina. La experiencia del Banco Nacional de Desarrollo 1967-1976. Bernal, UNQ.

Roze, J. (2007). Lucha de clases en el Chaco contemporáneo. Resistencia: Editorial La Paz.

Schaller, E. C. (2017). La tierra pública en el Chaco en los inicios del periodo provincial (19541971). $36^{\circ}$ Encuentro de Geohistoria Regional. Resistencia: IIGHI.

Viguera, A. (1998). Estado, empresarios y reformas económicas: en busca de una perspectiva analítica integradora. Revista Perfiles Latinoamericanos, 7 (12), 9-51. Recuperado de: http://perfilesla.flacso.edu.mx/index.php/perfilesla/article/view/379

Zurbriggen, C. (2006). El institucionalismo centrado en los actores: una perspectiva analítica en el estudio de las políticas públicas. Revista de Ciencia Política, Vol. 26, Núm.1. Recuperado de: http://www.redalyc.org/articulo.oa?id=324/32426104

\section{CURRICULUM VITAE}

\section{Adrián Alejandro Almirón}

Doctor en Humanidades y Artes con mención en Historia (UNR). Magister y Especialista en Ciencias Sociales con mención en Historia ( UNQ). Profesor Adjunto con Dedicación Exclusiva en la Cátedra de Historia Argentina y Latinoamericana Contemporánea de la Carrera de Comunicación Social, Facultad Humanidades.

almirón.historia@gmail.com 